\title{
Palm Print Recognition using Steerable Filter for Better Authentication System
}

\author{
Neha Dangi \\ Department of Computer Science \& Engineering \\ Oriental Institute of Science \& Technology, Bhopal
}

\author{
Atul Barve \\ Department of Information and Technology \\ Oriental Institute of Science \& Technology, Bhopal
}

\begin{abstract}
Every person has a unique palm print and by extracting the features from it; can build an authentication system likely fingerprint authentication. The proposed system is able to extract the ridge patterns present in the palm and compare it to the template stored in the data base and according to the matching key points it will authenticate a person. The acceptance rate of proposed palm prints authentication system is higher and also has high significant usability in the biometric identification technique. Some statistical properties will be compute which are necessary for the identification of an individual through the proposed system. The system uses some exceptional information from palm prints like principal lines, wrinkles, and textures while process of authentication carried out. Proposed methodology in the system tries to eliminate the probable deception that can't be evaded by the existing methods which uses identity cards and passwords. Proposed system offers high accuracy with null fraudulent for the identification purpose and can be widely used in many applications where authentication is the major part of processing the system.
\end{abstract}

\section{Keywords}

Palm Print, Authentication, Palm Crease, Gabor Filter, Biometric.

\section{INTRODUCTION}

Palm print authentication system is most reliable and effective biometric approach that can be utilizes in various fields. Palm print recognition is based on two lines first one is flexion that is also called principal lines and crease as secondary lines. On the basis of these two features, a palm print biometric system can be developed that possess ideal authentication system.

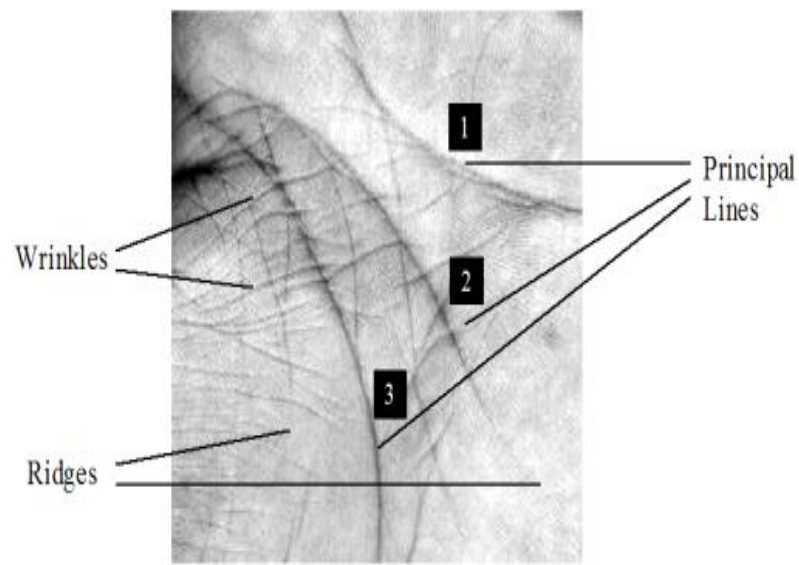

Fig. 1 Palm Features [5]

These lines are unique for every person, and it can help in forensic science where palm print has been found instead of fingerprint. Similarly, there are so many applications of palm print authentication system.

\section{RELATED WORKS}

Anil Singh Parihar et al. used Harris and SIFT methods to recognize palm print. This system uses only the middle part of a palm for unique crease measurements and later matching with trained database images. This system acquires some false acceptance rate which does not possess good authentication system. [1] Allen George et al. proposed a system that is based on few principal lines which are also known as flexion. Certain principal lines are thenar, hypothenar, and interdigital. This system has 5.7143 FRR (False Rejection Rate) which is very less as it should be. FRR means unauthorized users will not get access. More FRR means system has high proficiency to reject false users. [2] Indrayani Awate et al. proposed a system that is based on Canny edge detection method to detect edges presented on palm. In this system, the image of palm will be preprocessed for the enhancement of the image by the help of morphological technique. This system uses PolyU palmprint database. [3] Gaurav Jaswal et al. proposed a system that is based on texture information extracted from palm by using Gabor filter. In terms of calculating the difference between the images can be calculated by Euclidean distance between them. The accuracy of the system is an average of $83.12 \%$ which is bit lesser. [4]

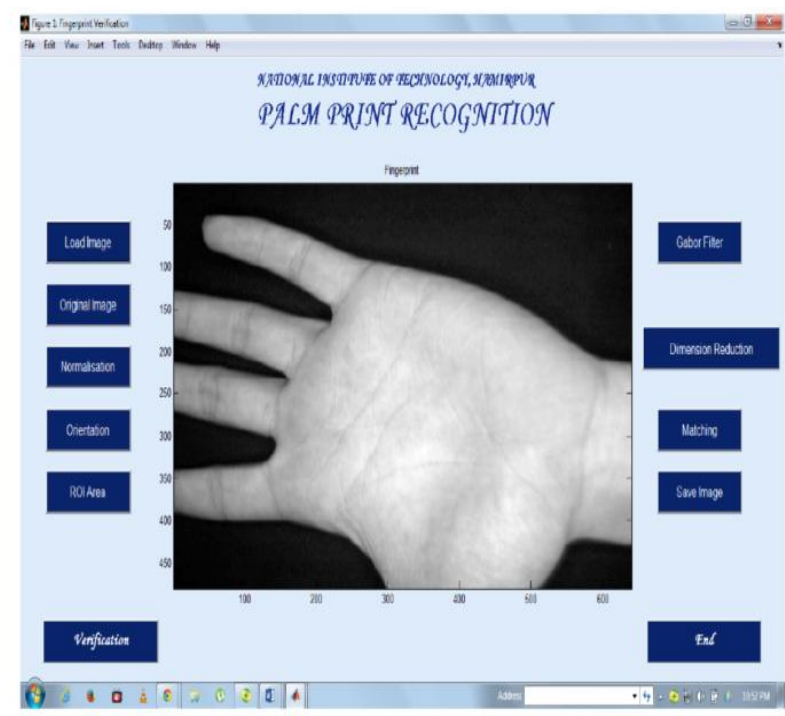

Fig.2 Graphical user Interface for Palm print Recognition [4]

Aishwarya D et al. proposed a system that is based on Weber's local descriptor algorithm to extract the information from palm. Euclidean distance formula has been used for calculating false rejection rate as well as false acceptance rate which calculates the accuracy of the system. System follows 
the principal lines, datum points, wrinkles and ridges. [5] Shivkant Kaushik et al. proposed a system that is based on hybrid approach for palm print recognition. Hybrid approach means that there are three image processing techniques have been used to extract the information from plam. This system also uses Gabor filter for preprocessing the data from palm image. In the phase of feature matching K-Nearest Classifier has been used. [6] Shalini Agrawal et al. proposed a system that is based on SVM classification method to generate feature vectors and experiment performed on polyU palm print database. This system possesses the ridge lines, wrinkles, principal lines for feature extracting and matching. Gabor filter has also been used for preprocessing the palm image for better outcomes. [7] Srijith Rajeev et al. proposed a system that is based on 3D depth information, 3-D technologies has made it easier to capture and store 3-D images. This system uses low pass filter for smoothing or denoising the palm print image. System uses 3D ROI instead of 2D ROI for mapping correct region of information that palm contains. [8]

\section{PROPOSED WORK}

Palmprint recognition system is one of the important features in the field of biometric systems, because it is distinct from the birth for every person even with twins, and also it has some advantages over the other signatures of biometrics like face recognition, iris recognition, voice recognition, etc. These advantages points can be listed as fixed line structure, easy and low cost, capturing and extracting of features even in low resolution imaging. Here, proposed system is able to extract the features with sharpen crease that lusted genuine biometric system. System extracted palm print feature and stored as template for feature matching. System uses steerable filter for extracting and refining palm print that enhances features acquisition for better and ideal system.

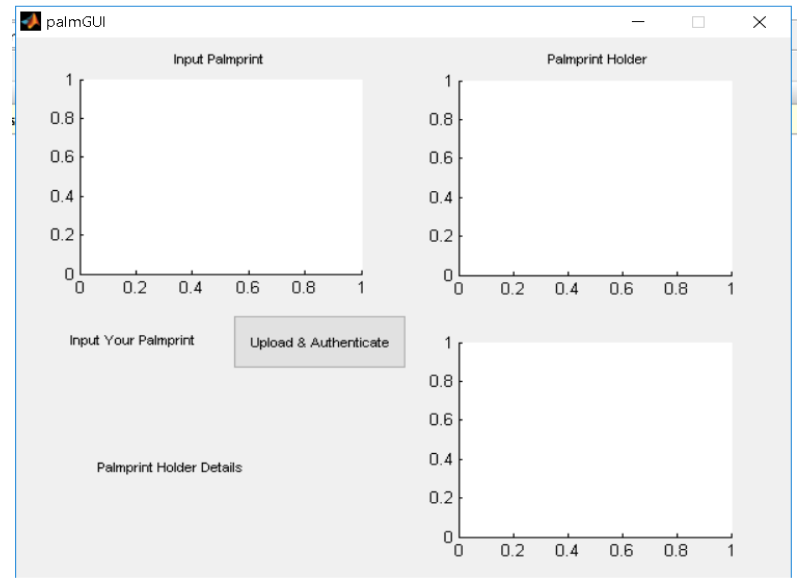

Fig. 3 Proposed System GUI

The system has been implemented in MATLAB with steerable filter that can upload a palm image and extract feature for matching with templates.

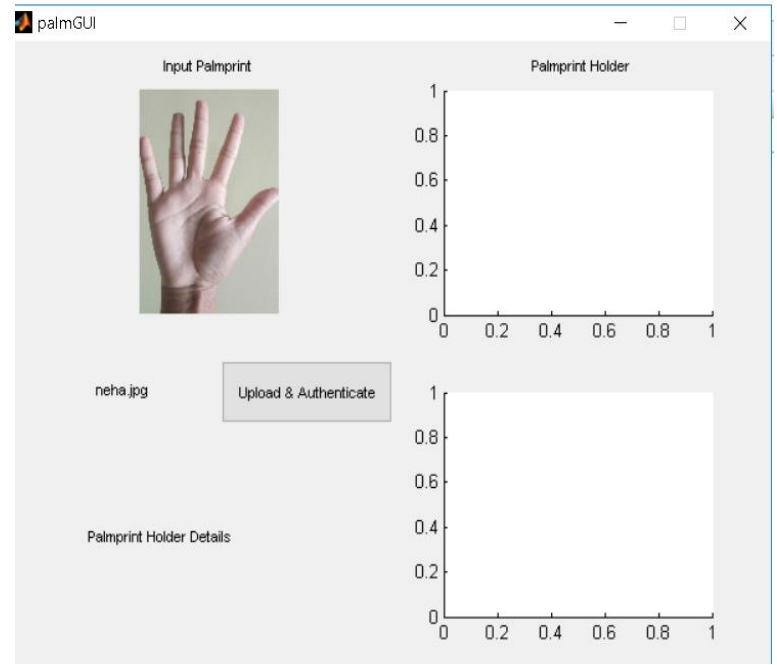

Fig. 4 Feature Extraction

Once the feature has been extracted, it matches with the stored templates and if the matching feature is greater than the threshold value or key points; the referential result will be returned with holder's detail. System is capable to return true positive results with minimal false or fake results. System has been tested with many enrollments and system observed as effective biometric authentication system. Recognizing Palm print to verify an individual is an authentic technique which can be employed for the verification process. Palm consists of various unique features like wrinkles and principal lines, through which huge amount of information can be extracted to distinctively verify a person. By examine the extracted images of palm print, it is comprises of various characters-

- The value of intensity obtained from palm lines is lower than surfaces.

- Gray scale values of Principal lines are lower than that of wrinkles.

- There are irregular shapes, directions and depth of every palm lines.

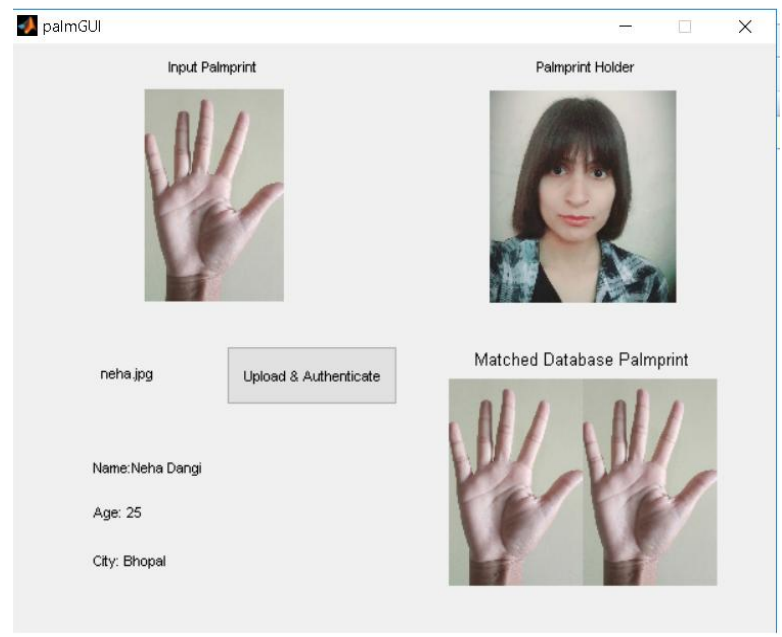

Fig. 5 Feature Matching 

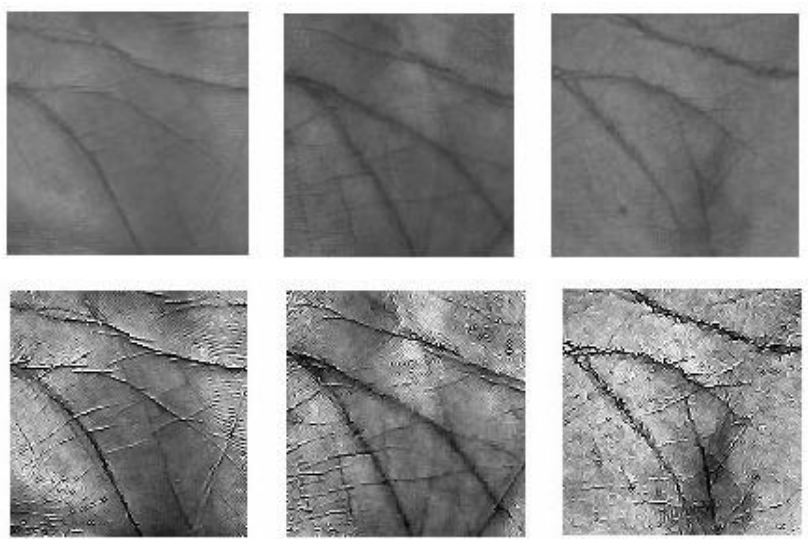

Fig.6 Steerable Sharpen Features

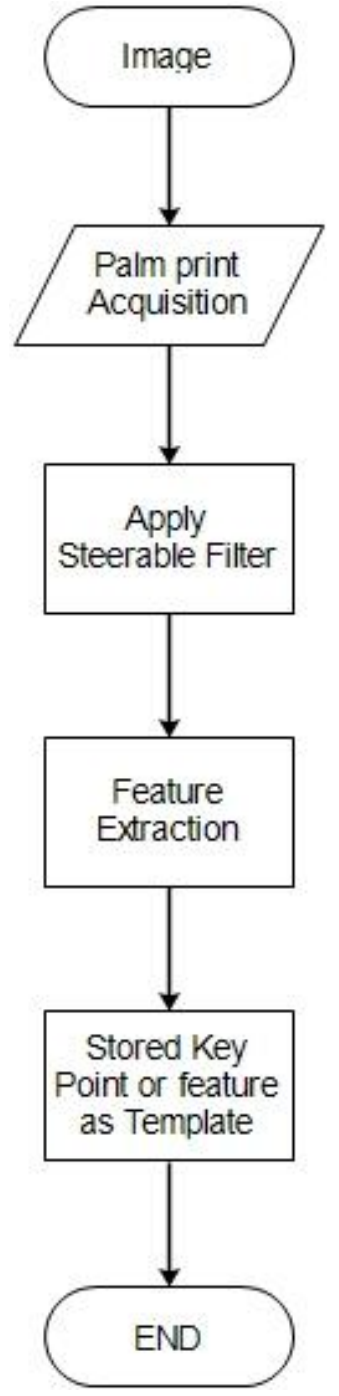

Fig. 7 Flow Chart for Feature Template

Fig. 3.5 shows the enrollment process that firstly acquire a palm image then system refines palm print from image and apply steerable filter for achieving it. Once the features have been extracted from palm print image it stored as template for template matching. When a palm image has been given for authentication, system started achieving its features and comparing with stored templates. If matching key points are greater than the decided threshold value it will successfully authenticate the user and display stored details but if it is less than threshold value then authentication will be denied and no result will be displayed over there.

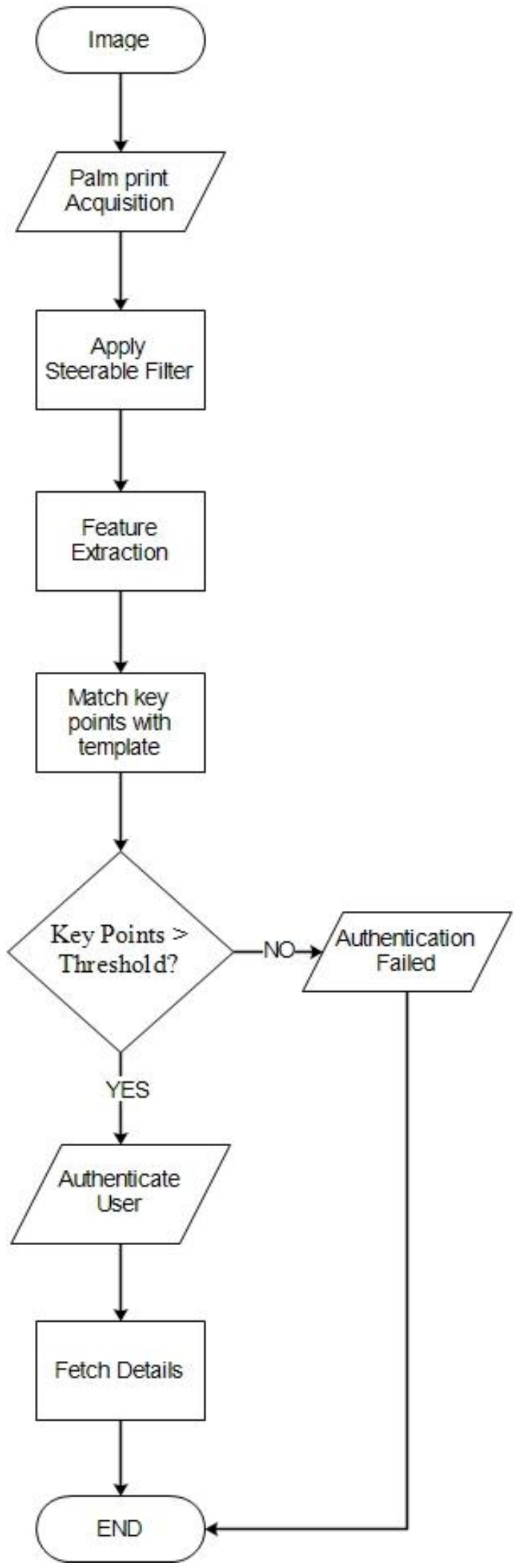

Fig. 8 Flow Chart for Feature Extraction and Matching

Some high pass filters like Laplacian filter was exploited earlier to acquire the components of high frequency and effectively drawn attention on the feature but the used approach was very sensitive to noise. So in order to reduce 
noise and to provide components of high frequency, steerable filters are exploited in the system that too in various directions. Steerable filters are basically a form of oriented filters; it means a filter which is responsive at every point of reference. These filters are applied easily to reduce the noise of an image, detect features and segment the colors. It is a kind of filter in which filter that consists of subjective direction is synthesized.

Let it be more precise with an algorithm.

\subsection{SF (Steerable Filter) Algorithm:}

Require: $I$ as input palm image, $\mathrm{G}$ as Gaussian function, $\mathrm{x}$ and $\mathrm{y}$ as Cartesian coordinates, $\theta$ as rotation angle, $\cos (\theta)$ and $\sin (\theta)$ as corresponding interpolation function, $A_{n}$ as stored data from database.

Input: $I$ as palm input image.

Output: $R_{1}^{\theta}$ as filtered image derivative

1: Palm image acquisition

2: Apply steerable filter to extract the features, initialize Gaussian function

$$
\mathrm{G}(x, y)=e^{-\left(x^{2}+y^{2}\right)}
$$

where $\mathrm{G}$ is a Gaussian function and $\mathrm{x} \& \mathrm{y}$ are Cartesian coordinates

3: Declare rotation operator $(\ldots)^{\theta}$ for any function

$f^{\theta}(x, y)$ that rotated through an angle $\theta$ about the origin

4: Evaluate the first $x$ derivative of a Gaussian

$G_{1}^{0^{\circ}}=\frac{d}{d x} e^{-\left(x^{2}+y^{2}\right)}=-2 x e^{-\left(x^{2}+y^{2}\right)}$

$G_{1}^{90^{\circ}}=\frac{d}{d x} e^{-\left(x^{2}+y^{2}\right)}=-2 y e^{-\left(x^{2}+y^{2}\right)}$

$$
G_{1}^{\theta}=\cos (\theta) G_{1}^{0^{\circ}}+\sin (\theta) G_{1}^{90^{\circ}}
$$

$\cos (\theta)$ and $\sin (\theta)$ are corresponding interpolation function

5: Apply parameters where $I$ is an input palm image

$$
\begin{gathered}
R_{1}^{0^{\circ}}=G_{1}^{0^{\circ}} * I \\
R_{1}^{90^{\circ}}=G_{1}^{90^{\circ}} * I \\
R_{1}^{\theta}=\cos (\theta) R_{1}^{0^{\circ}}+\sin (\theta) R_{1}^{90^{\circ}}
\end{gathered}
$$

where $R_{1}^{\theta}$ is an filtered image derivatives

6: if $R_{1}^{\theta}>$ threshold value then

Print $\left\{A_{n}\right.$ w.r.t. acquired data set $\}$

else

Print $\{$ Default denied message $\}$

end else

end if

\section{7: End}

\section{RESULT ANALYSIS}

The system has been tested with 25 users along with different palm prints. There are 24 true positive achieved and 1 true negative, it means that 24 palm prints get accessed successfully but proposed system missed one of them. That affected the precision rate of the system. System also tested with those palm print which are not present in database that should be rejected while authenticating them. System is able to reject false users with high level of accuracy because if an unauthorized user get accessed that possesses system failure. If a true is rejected then system does not retain machine failure but one false acceptance will reject the authentication

\begin{tabular}{|c|c|c|c|c|}
\hline Users & $\mathbf{T P}$ & $\mathbf{T N}$ & FP & FN \\
\hline 1 & 1 & 0 & 0 & 0 \\
\hline 2 & 1 & 0 & 0 & 0 \\
\hline 3 & 1 & 0 & 0 & 0 \\
\hline 4 & 1 & 0 & 0 & 0 \\
\hline 5 & 1 & 0 & 0 & 0 \\
\hline 6 & 1 & 0 & 0 & 0 \\
\hline 7 & 1 & 0 & 0 & 0 \\
\hline 8 & 1 & 0 & 0 & 0 \\
\hline 9 & 1 & 0 & 0 & 0 \\
\hline 10 & 1 & 0 & 0 & 0 \\
\hline 11 & 1 & 0 & 0 & 0 \\
\hline 12 & 1 & 0 & 0 & 0 \\
\hline 13 & 0 & 1 & 0 & 0 \\
\hline 14 & 1 & 0 & 0 & 0 \\
\hline 15 & 1 & 0 & 0 & 0 \\
\hline 16 & 1 & 0 & 0 & 0 \\
\hline 17 & 1 & 0 & 0 & 0 \\
\hline 18 & 1 & 0 & 0 & 0 \\
\hline 19 & 1 & 0 & 0 & 0 \\
\hline 20 & 1 & 0 & 0 & 0 \\
\hline 21 & 1 & 0 & 0 & 0 \\
\hline 22 & 1 & 0 & 0 & 0 \\
\hline 23 & 1 & 0 & 0 & 0 \\
\hline 24 & 1 & 0 & 0 & 0 \\
\hline 25 & 1 & 0 & 0 & 0 \\
\hline Total & 24 & 1 & 0 & 0 \\
\hline
\end{tabular}
system and system becomes useless. The precision rate of proposed system is bit higher as compare to the earlier one with minimal error or false acceptance rate.

Table No. 1 Result Analysis

TP- True Positive, TN-True Negative, FP-False Positive, FNFalse Negative

$$
\text { Precision }=\frac{T P}{T P+T N+F P} * 100 \%
$$




$$
\text { Precision }=\frac{24}{24+1+0} * 100 \%
$$

Precision $=96 \%$

Table No. 2 Result Comparison

\begin{tabular}{|l|l|l|}
\hline SCHEME & PRESENT & PROPOSED \\
\hline $\begin{array}{l}\text { Total no. of palm } \\
\text { image tested }\end{array}$ & Not Mentioned & 25 \\
\hline $\begin{array}{l}\text { No. of false palm } \\
\text { image tested }\end{array}$ & - & 8 \\
\hline $\begin{array}{l}\text { No. of true palm } \\
\text { image tested }\end{array}$ & - & 17 \\
\hline $\begin{array}{l}\text { False Recognition or } \\
\text { Missed by algorithm }\end{array}$ & - & 1 \\
\hline Precision & $94.5 \%$ & $96 \%$ \\
\hline
\end{tabular}

\section{CONCLUSION \& FUTURE SCOPE}

Thus the system which has been proposed is able to provide better level of precision as compare to the earlier implemented system. Here system has been implemented with steerable filters instead of Gabor filter which has been used by previous system. Steerable filter is able to find edges by rotating the image orientation with different angles that retains sharp palm crease. Here, the precision rate of proposed system is $96 \%$ along with zero false acceptance rates. System can be implemented with higher level of accuracy in future that can be applicable in various fields of biometric authentication system.

\section{REFERENCES}

[1] Anil Singh Parihar1, Amoiy Kumar2, Om Prakash Verma3, Ankita Gupta4, Prerana Mukherjee5 , and Deepika Vatsa6 "Point Based Features for Contact-less Palmprint Images", IEEE Transaction, 2013.

[2] Allen George, G.Karthick, Dr.R.Harikumar, "An Efficient System for Palm Print Recognition using Ridges”, IEEE Transaction, 2014.

[3] Indrayani Awate and Prof. B.A. Dixit, "PALM PRINT BASED PERSON IDENTIFICATION", IEEE Transaction, 2015.

[4] Gaurav Jaswal, Ravinder Nath, Amit Kaul, "Texture based Palm Print Recognition using 2-D Gabor Filter and Sub Space Approaches", IEEE Transaction, 2015.

[5] Aishwarya D, Gowri M Saranya R K, "Palm Print Recognition Using Liveness Detection Technique", IEEE Transaction, 2016.

[6] Shivkant Kaushik1, Rajendra Singh2, "A New Hybrid Approch for Palm Print Recognition in PCA Based Palm Print Recognition System”, IEEE Transaction, 2016.

[7] Shalini Agarwal, Pawan Kumar Verma and Mohd Aamir Khan "An Optimized Palm Print Recognition Approach using Gabor filter”, IEEE Transaction 2017.

[8] Srijith Rajeev and Sos Agaian, "3-D Palmprint Modeling for Biometric Verification”, IEEE Transaction 2017.

[9] http://academyofhandanalysis.org/tag/handprinting/page/3/ 